\title{
Cosecha ganglionar en cáncer de colon. Mitos y magia
}

Ricardo Misa*, Mario Almada†, Noelia Brito ${ }^{\ddagger}$ José Martínez ${ }^{\S}$, Andrés Pouy ${ }^{\Uparrow}$, Camila Haro**

\section{Resumen}

El compromiso ganglionar es crítico en la estadificación del cáncer de colon como factor pronóstico y como determinante de tratamiento adyuvante. Se sigue discutiendo el número de ganglios adecuados a resecar, cuáles son los factores que inciden en la cosecha ganglionar y el significado biológico de ésta. Se revisan las variables clínicas y de la propia biología tumoral que hacen que la definición de un número determinado de ganglios, como gold standard de cosecha ganglionar adecuada, sea controversial. El número 12 no necesariamente es un número "mágico" marcador de calidad. Extender la resección para aumentar la cosecha ganglionar no mejora la estadificación, expone al paciente a riesgos innecesarios, sin efecto terapéutico comprobado. La "magia" sigue siendo realizar resecciones regladas, que incluyan el pedículo vascular y el meso satélite al tumor, ajustando la resección a las características del paciente. Menos no es más, pero más no es necesariamente mejor.

Palabras clave: Neoplasias del colon Estadificación de neoplasias

Cosecha ganglionar

Key words: Colonic neoplasms Neoplasms stading Lymph node harvest
Mito: "Persona o cosas a las que se atribuyen cualidades o excelencias que no tienen, o bien una realidad de la que carecen".

Magia: "La que por medios naturales obra efectos que parecen sobrenaturales".

\section{Introducción}

Desde Dukes hasta la fecha, el pronóstico del cáncer de colon no metastásico se relaciona con el grado de infiltración en la pared y a la presencia o ausencia de enfermedad ganglionar ${ }^{(1)}$.

La definición del compromiso ganglionar en el cáncer de colon es un componente crítico en la estadificación y es el factor pronóstico más importante en el cáncer colónico sin enfermedad sistémica ${ }^{(2)}$.

Agregado a esto, la presencia de nódulos ganglionares metastásicos determina la utilización de terapia adyuvante, que ha demostrado en forma inequívoca el aumento de sobrevida y el intervalo libre de enfermedad en los pacientes en estadio III.

El metaanálisis de Bockelman, con más de 36.000 pacientes incluidos, en estudios de muy buena calidad, muestra un intervalo de sobrevida libre de enfermedad (ISL) de $82,7 \%$ para pacientes en estadio II sin coadyuvancia. En el estadio III, el intervalo libre de enfermedad sin coadyuvancia cae $49 \%$, mejorando hasta $63,8 \%$ con la utilización de quimioterapia adyuvante ${ }^{(3)}$.

La objetivación de metástasis ganglionares es el predictor más fuerte en el cáncer de colon sin enfermedad sistémica $^{(4-6)}$.

Asumir la importancia de la enfermedad metastásica ganglionar y su implicancia, tanto pronóstica como terapéutica, provocó la aparición de numerosas interro-

\footnotetext{
* Prof. Agdo. Clínica Quirúrgica "B”, Hospital de Clínicas. Uruguay.

† Asist. Clínica Quirúrgica "B", Hospital de Clínicas. Uruguay.

$\ddagger$ Asist. Clínica Quirúrgica "B", Hospital de Clínicas. Uruguay.

$\S$ Ex residente Clínica Quirúrgica "B”, Hospital de Clínicas. Uruguay.

ๆ Residente Clínica Quirúrgica "B”, Hospital de Clínicas. Uruguay.

** Residente Clínica Quirúrgica "B", Hospital de Clínicas. Uruguay.

Clínica Quirúrgica "B" Prof. Dr. C. Canessa.

Los autores no presentan conflicto de intereses.

Correspondencia: Ricardo Misa. Pablo Podestá 1425, Montevideo, Uruguay. Correo electrónico: rmisa@adinet.com.uy

Recibido: $7 / 10 / 19$

Aprobado: $11 / 12 / 19$
} 


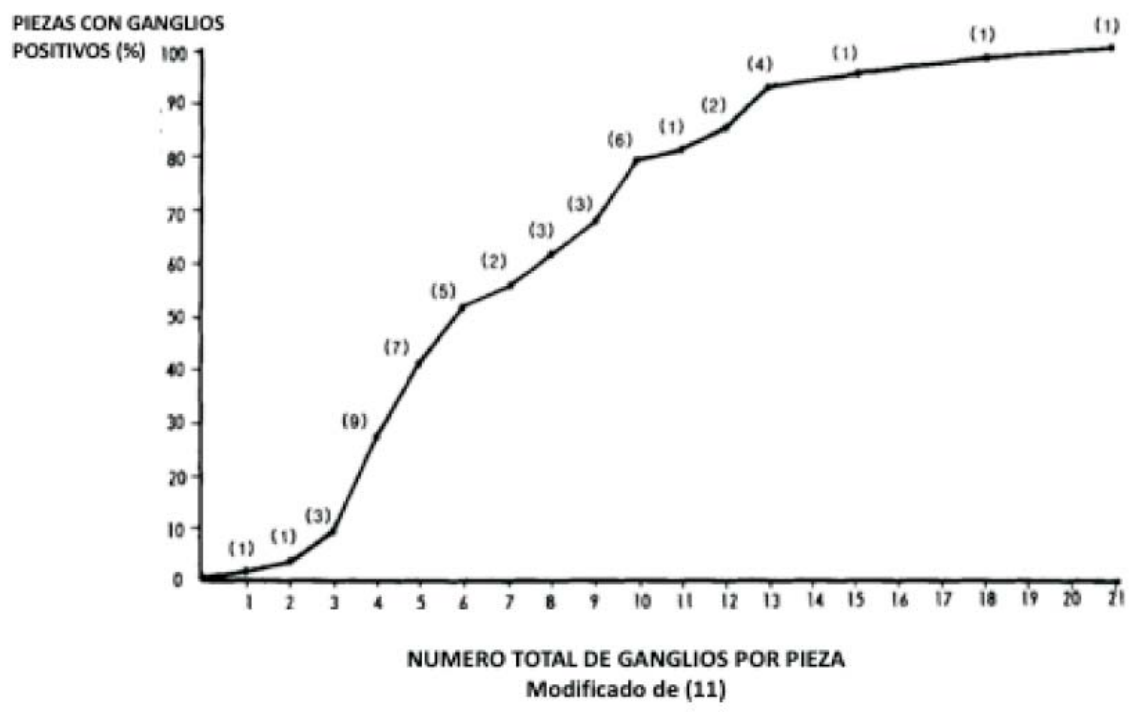

Figura 1. La posibilidad de identificar ganglios positivos es mayor a medida que se identifica un mayor número de ganglios. (Modificado de Scott ${ }^{(11)}$ ).

gantes y controversias que se mantienen hasta el día de hoy $^{(7-8)}$ :

- ¿Cuál es el número mínimo de ganglios que deben ser analizados para definir un M0?

- ¿Cuáles son los factores de confusión?

- ¿Tiene la linfadenectomía efectos terapéuticos? ¿Se justifican las resecciones extendidas? ¿Más es mejor?

- ¿Por qué los estudios muestran correlación entre el conteo ganglionar y la sobrevida?

- ¿Cuál es el significado biológico de la cosecha ganglionar?

\section{El 12 como "número mágico"}

La importancia pronóstica y terapéutica de la identificación del compromiso ganglionar trae aparejado un problema que no es menor. ¿Cuál es el número mínimo de ganglios que deben ser resecados e identificados para una correcta estadificación?

Este número se conoce también como cosecha ganglionar y aquí tenemos el primer punto controversial.

Es lógico pensar que una cosecha ganglionar pobre llevará en primer lugar a una subestadificación, por lo tanto privará del beneficio de la terapia coadyuvante, por existencia de metástasis ganglionares no evidenciadas y, por otra parte, a la existencia de enfermedad mesial residual, causante de recaída tumoral ${ }^{(4)}$.

En $1991^{(9)}$ se publica un documento de consenso elaborado en el Congreso Mundial de Gastroenterología de 1990 en Sidney, donde se establece que "antes de considerar una resección radical sin metástasis gangliona- res, se recomienda examinar al menos 12 ganglios linfáticos. Si 12 nodos no son identificados por los métodos tradicionales, se deben considerar métodos adicionales de disección".

Esta recomendación se basó en trabajos como el de Pickren, y especialmente en el de $\operatorname{Scott}^{(10,11)}$, que por métodos de aclaramiento graso señalaban la mayor posibilidad de estadificar como estadio ganglionar más avanzado a medida que se aumentaba el número de ganglios examinados (figura 1).

Estos trabajos tenían un nivel de evidencia que no era óptimo:

- Trabajos débiles desde el punto de vista estadístico, con muestras bajas y resultados que no tomaban en cuenta distintas variables de confusión.

- Opiniones de expertos.

Esta recomendación fue adoptada por la AJCC American Joint Comittee on Cancer (AJCC), el Colegio de Patólogos Estadounidenses y la Red Integrada de Cáncer $(\mathrm{NCCN})^{(6)}$. Recomiendan por unanimidad la evaluación de tantos ganglios linfáticos como sea posible, con un mínimo de 12 ganglios linfáticos en total por espécimen, para garantizar resecciones rigurosas y evitar falsos negativos ${ }^{(12)}$.

El número 12 quedó asociado a calidad de la cirugía, a calidad del examen patológico y por lo tanto a calidad del centro quirúrgico ${ }^{(13)}$.

Parsons ${ }^{(14)}$, en un análisis de las cosechas ganglionares en Estados Unidos entre 1998 y 2009, en más de 
90.000 pacientes, encuentra una adhesión progresiva a las guías a medida que las distintas asociaciones científicas se adherían a las mismas, con $34 \%$ de pacientes con más de 12 ganglios en los años 90 a $75 \%$ de pacientes con más de ese número a partir de 2009.

Actualmente, Backes ${ }^{(15)}$ también encuentra en un análisis de tumores T1 una diferencia de $20 \%$ a $66 \%$ en cosechas ganglionares mayores a 10 de acuerdo a cirugías realizadas antes o después de 2010.

Para el patólogo, el problema principal es el "techo ganglionar" ${ }^{(13)}$, es decir, hasta cuándo tiene que buscar o utilizar técnicas más complejas (de aclaramiento ganglionar) para lograr el número adecuado para la estadificación ${ }^{(16)}$. Este problema es todavía más complejo si atendemos a los trabajos actuales que dan mayor importancia al radio ganglionar, o sea, a la relación entre el número de ganglios metastasiados y el total de ganglios de la cosecha ganglionar ${ }^{(17)}$.

Para el cirujano, la visión es distinta. No se trata de un problema económico o de tiempo, sino de enfrentar beneficios de resecciones ganglionares mayores versus cirugías extendidas con mayor morbilidad.

El objetivo de los 12 ganglios linfáticos es actualmente un requisito para la certificación por el Colegio Americano de Cirujanos. No es sorprendente que las compañías de seguros hayan sido rápidas en la implementación de este requisito para negar el pago, aduciendo la ausencia de calidad en la cirugía realizada ${ }^{(18)}$.

$\mathrm{Liu}^{(19)}$, en un análisis de las medidas para el control de calidad del Colegio Americano de Cirujanos (ACS), llama la atención sobre la confiabilidad del número 12 especialmente en períodos cortos de tiempo, para evaluar la calidad de la cirugía, y resalta que el uso de medidas que no entregan evaluaciones confiables, especialmente a nivel del proveedor, podrían generar esfuerzos de mejora de rendimiento inadecuados, o, lo que es peor, resultar en sanciones o cambios en los reembolsos que son infundados y dificultan la atención del paciente.

La definición de 12 como gold standard para definir una cosecha ganglionar como adecuada es controversial y para muchos arbitraria, ya que existen factores no dependientes del cirujano y el patólogo que pueden llevar a la variación de aquélla ${ }^{(20,21)}$.

\section{¿De qué depende la cosecha ganglionar? ¿Una cirugía de calidad debe obtener siempre 12 ganglios o más?}

Ya lo hemos mencionado, y toda la literatura es consistente en que la extensión de la resección visceral y mesial es el primer factor que incide en la cosecha ganglionar $^{(5,8,22)}$. La extensión de la resección y el análisis patológico correcto son los factores modificables. Pero existen una serie de factores independientes, propios del pa- ciente y del tumor, que se agrupan desde el punto de vista metodológico como factores de confusión y que son responsables de las controversias sobre el resultado adecuado del rendimiento ganglionar ${ }^{(13,23)}$.

\section{Variables clínicas}

Las variables clínicas que se han analizado son la edad, el índice de masa corporal (IMC), el ASA (American Society of Anesthesiologists), el sexo y las condiciones socioeconómicas y raza del paciente.

Las tres últimas son discutibles y se asocian más a factores demográficos y acceso a la salud.

Con respecto a la obesidad, parecería que disminuye el rendimiento ganglionar. Se invoca, por un lado, el deterioro inmunológico $\mathrm{y}$, por otro, las dificultades quirúrgicas vinculadas a la resección, pero los resultados en la bibliografía son disímiles ${ }^{(23)}$.

La edad es el factor propio del paciente con mayor peso en la variación ganglionar. Esta variable tiene dos aspectos: por una parte, es lógico pensar que en los pacientes añosos las resecciones tienden a ser más económicas.

Por otro lado, se asocia la inmunosenescencia propia de estos pacientes. Algunos trabajos señalan un descenso en el rendimiento ganglionar de casi $7 \%$ cada diez años de incremento de edad ${ }^{(21,24)}$.

Con el ASA seguramente exista una relación similar a lo que ocurre con la edad, vinculado a resecciones menos extensas y a la presencia de inmunocompromiso ${ }^{(23)}$.

También se ha señalado que en la cirugía de urgencia es de esperar cosechas ganglionares más pobres (cirujanos menos entrenados, cirugías abreviadas), aunque los trabajos son contradictorios y algunos no muestran diferencias $^{(25,26)}$.

\section{Variables propias del tumor ${ }^{(13,23)}$}

Tamaño: está señalado que los tumores más grandes provocan más inmuno reacción, especialmente si hay necrosis. Varios trabajos señalan que por cada centímetro de tumor aumenta en un $2 \%$ la posibilidad de cosechas ganglionares adecuadas.

Presencia de infiltración linfocitaria: a mayor infiltración linfocitaria, mayor conteo ganglionar ${ }^{(27)}$.

Grado de diferenciación: el conteo ganglionar adecuado es más frecuente en los tumores pobremente diferenciados y seguramente hay varios factores que lo explican, como el mayor tamaño tumoral y la presencia de metástasis ganglionares más tempranas

Presencia de inestabilidad microsatelital (IMS): la IMS define al tumor con menor agresividad, pero paradojalmente tiene una mayor respuesta inflamatoria y es más frecuente cosechas ganglionares. 
Localización: los tumores de colon derecho se asocian a conteos ganglionares mayores y existen varias causas que lo pueden explicar. Por un lado, existe una mayor extensión de resección visceral y mesial reglada, lo que puede no reproducirse en los tumores izquierdos, donde las resecciones sectoriales son aceptadas.

Además, existen anatómicamente más estructuras ganglionares a lo largo de los vasos mesentéricos superiores.

A esto se agrega que el colon derecho es la localización preferente para tumores con inestabilidad satelital.

La recomendación actual es, entonces, para algunos autores demasiado rígida, poco individualizada, y que no toma en cuenta las características particulares del paciente y del propio tumor ${ }^{(20,21)}$

Todavía no existe en la literatura uniformidad en determinar cuál es el número mínimo de ganglios que permita asegurar la ausencia de subestadificación, pero cada vez más aparecen autores que llaman la atención frente a la rigidez quirúrgica para lograr el "número adecuado" ${ }^{\text {(13,28) }}$. Muchos autores están de acuerdo en que hay que tomar en cuenta la variabilidad del número y no tomarlo como un indicador aislado ${ }^{(22)}$.

A esto se agrega otro problema interesante: toda la literatura actual coincide en que la sobrevida del cáncer de colon está asociada en forma independiente con el número de ganglios analizados al tiempo de la colectomía ${ }^{(6)}$. Numerosos estudios han mostrado que el incremento en el número de ganglios resecados mejora la sobrevida tanto en pacientes con nódulos ganglionares positivos o negativos $^{(6,29-31)}$. Esto ha llevado a la promoción de resecciones más extensas, que, como veremos más adelante, es motivo de fuertes controversias.

\section{La controversia: ¿más es mejor?}

Los trabajos que abogan a favor de las resecciones extensas parten del concepto de que el rendimiento ganglionar parecería ser un factor pronóstico independiente. En el estadio II ligado a mayor seguridad en la estadificación y en el estadio III asociado a mayor clearance tumoral $^{(32)}$.

El concepto básico para estos autores sería: si la cosecha ganglionar es pobre, la posibilidad de subestadificar es mayor y el número de ganglios metastasiados aumenta a medida que aumenta la cosecha ganglio$\operatorname{nar}^{(33,34)}$

Otro mecanismo invocado es que la linfadenectomía extendida, en el entorno de una escisión meso colónica completa, permitiría eliminar las "metástasis saltarinas". Si bien varios estudios señalan esa posibilidad, todavía falta evidencia concluyente.
Otros trabajos también van en esta dirección. Choi ${ }^{(35)}$ señala que deberían examinarse al menos 21 ganglios para asegurar una correcta estadificación E II.

$\mathrm{O}^{\prime}$ Boyle ${ }^{(36)}$ redobla la apuesta, encontrando una mayor sobrevida e intervalo libre de enfermedad cuando las cosechas ganglionares son mayores a 21 nodos.

Ahora bien, la constatación de que el pronóstico del cáncer tiene relación independiente con el rendimiento ganglionar ha llevado también a la aparición de trabajos que a nuestro juicio tienen conclusiones erróneas o que deben ser analizadas con cautela.

Gravante ${ }^{(37)}$, analizando el rendimiento ganglionar y su relación con la longitud de la resección, encuentra que las amputaciones abdómino perineales y la operación de Hartmann no tienen frecuentemente un rendimiento ganglionar adecuado, y concluyen que en estos pacientes deben realizarse siempre maniobras, como el descenso del ángulo izquierdo de forma que se asegure una mayor resección y un rendimiento ganglionar "adecuado".

Stracci ${ }^{(38)}$ también apoya la realización de resecciones extensas para asegurar el correcto rendimiento ganglionar.

Khan ${ }^{(39)}$ promueve hacer esfuerzos para extender la resección en pacientes de la tercera edad, ya que éstos llevarían a subestadificación y a no realizar coadyuvancia.

Lee ${ }^{(40)}$ señala que debido al peor pronóstico del cáncer de colon derecho, y al hecho de que el alto rendimiento ganglionar se asocia con mayor sobrevida, puede ser recomendable en estos pacientes realizar una estrategia de escisión mesocolónica completa.

Sin embargo, muchos trabajos no están de acuerdo con esta postura, poniendo en duda que la extensión de la resección visceral y mesial sean los responsables del aumento de la recidiva.

En 2014, van Erning ${ }^{(27)}$, realizando un análisis de la tendencia en cosechas ganglionares en diez años (en pacientes con cáncer de colon M0), encuentra que el aumento en las cosechas ganglionares no se acompañó de un aumento en el hallazgo de nodos positivos para tumor, concluyendo que si bien existía una relación entre el rendimiento ganglionar y la sobrevida, esta no dependía de la subestadificación.

Parsons ${ }^{(41)}$ refiere resultados similares. En un estudio observacional sobre una base de datos con más de 86.000 pacientes encontró que a pesar de que se incrementó marcadamente en las últimas dos décadas el número de ganglios evaluados, esto no se asoció con un aumento en la cantidad de ganglios positivos y, por lo tanto, no existió incremento en la estadificación.

Budde ${ }^{(42)}$ realiza una revisión similar sobre una base de datos de más de 147.000 pacientes. Encuentra que el 


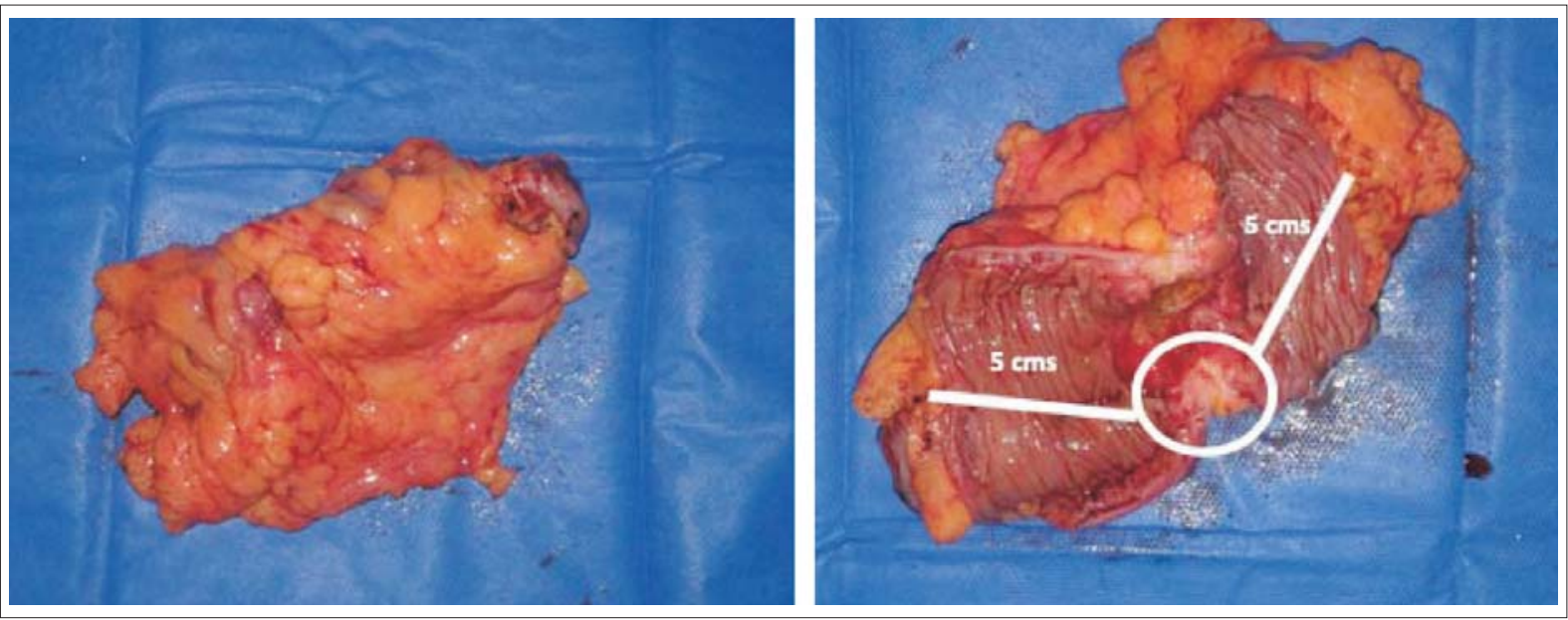

Figura 2. Resección segmentaria. Zona de mayor incidencia de adenopatías positivas según Lisovsky ${ }^{(4)}$.

número de ganglios analizados se incrementó secuencialmente de 12 en 2004 a 17 en 2010, pero el aumento en el rendimiento ganglionar no se acompañó de un aumento en los estadios III.

Del Paggio ${ }^{(43)}$ realiza una revisión de historias clínicas electrónicas entre 2002 y 2008, con algo más de 5.500 pacientes con cáncer de colon. Señala que a pesar de que existe un aumento en los porcentajes de cosecha ganglionar, el porcentaje de pacientes con ganglios positivos sigue siendo similar: $53 \%$ en 2008 vs $54 \%$ en 2002.

Kawamura ${ }^{(44)}$ realiza una revisión retrospectiva de las anatomías patológicas de más de 500 pacientes con cáncer de colon, separando dos grupos: uno con ligaduras altas y otro con ligaduras bajas, de acuerdo al sitio de ligadura de los vasos de drenaje.

Para el ciego, colon ascendente y transverso, la ligadura alta implicaba la ligadura en la raíz de la íleo cólica, la cólica derecha y la media (o de ambas) y la ligadura baja significaba la ligadura a nivel de la propia rama principal.

Para el colon izquierdo, la ligadura alta fue definida como la ligadura de la mesentérica inferior en la raíz, y la ligadura baja como la ligadura de la cólica izquierda, o de la rectal superior.

Señala que en el $90 \%$ de sus pacientes el compromiso ganglionar fue limitado a los ganglios pericólicos. Por lo tanto, la ligadura alta para una resección mayor no tiene beneficios y sí trae problemas. Las recidivas no se producen necesariamente en el meso y existen todos los inconvenientes de la ligadura alta.

Varios trabajos de disección anatómica, realizados por cirujanos o patólogos, también van en esta dirección.
Canessa $^{(45)}$, en un trabajo cadavérico, encuentra en disecciones ganglionares de meso recto, un promedio de 8,4 ganglios por pieza, con una media de 7 , señalando que la existencia de rangos de variación en el número de ganglios es habitual y debe ser tenido en cuenta cuando se realiza esta valoración.

En esta misma línea, Hashiguchi ${ }^{(46)}$ muestra un trabajo con más de 900 pacientes con cáncer de colon donde mapeó los ganglios en tres grupos: horizontales (epi y paracólicos), intermedios (ganglios meso cólicos) y ganglios en el tronco arterial principal. El autor encuentra que la resección de las adenopatías troncales no aumentó la estadificación, ni mejoró la sobrevida.

Lisovsky $^{(4)}$ encuentra que los ganglios positivos están habitualmente en el meso hasta $5 \mathrm{~cm}$ del límite del tumor para proximal y distal. Solo en cuatro pacientes de 46 había ganglios positivos en el tejido linfo ganglionar más allá de los $5 \mathrm{~cm}$, pero en los cuatro casos había también ganglios en el tejido linfo ganglionar satélite a los primeros $5 \mathrm{~cm}$ (figura 2).

Estos hallazgos argumentan en contra de los resultados obtenidos en otros centros con las escisiones meso colónicas totales (EMC) y las ligaduras vasculares centrales. La EMC expone órganos y vasos que usualmente no son puestos en riesgo por la cirugía "convencional", por lo que existe importante morbimortalidad ligada al procedimiento $^{(6,47)}$.

Estos mismos conceptos son manejados desde distintos ángulos por otros autores:

$\mathrm{Fan}^{(48)}$, comparando ligadura vascular por debajo de la cólica izquierda o a nivel de la raíz de la mesentérica inferior, en cáncer sigmoideo o rectal, encuentra que en los pacientes con ligaduras radicales existe un aumento en las fallas de sutura, sin aumento de los ganglios positivos obtenidos con dicha ligadura alta. 
Paquette ${ }^{(49)}$ realiza una revisión bibliográfica de más de 180 artículos que analizan la resección en nivel D3 o la EMC.

Este autor señala que las metástasis a nivel de los nódulos centrales se observan entre el 1\% y $8 \%$ de los pacientes con cáncer de colon, y esto ocurre en pacientes con tumores T3 o T4. Estos nódulos centrales positivos se asocian con la disminución de la sobrevida y deben considerarse como metástasis sistémicas.

Cotte ${ }^{(50)}$, en el análisis de la calidad oncológica de la colectomía angular en esta situación se pregunta: ¿cuál es la utilidad de incluir en la resección un territorio de drenaje linfático contralateral? Y señala: "La colectomía subtotal en esta situación sacrifica inútilmente una gran parte del colon, aumentando las secuelas funcionales, sin beneficio oncológico". Agregamos que además se expone al paciente a mayor disección quirúrgica, con posibilidad de lesiones y complicaciones que no se verían frente a una cirugía segmentaria.

\section{¿Por qué los estudios muestran correlación entre el conteo ganglionar y la sobrevida? ¿Cuál es el significado biológico de la cosecha ganglionar?}

Si bien los trabajos mencionados previamente ${ }^{(12,27,41-43)}$, señalan que el mayor rendimiento ganglionar no se acompaña de aumento de positividad ganglionar, en todos se objetiva una mejoría en la sobrevida a medida que aumenta el rendimiento ganglionar.

Esto lleva a poner en duda que el mejor pronóstico de los pacientes con cosecha ganglionar alta sea por disminuir la posibilidad de subestadificación, sino que lo que habla es de una mejor respuesta inmune. El hecho de que la inestabilidad satelital y los pacientes jóvenes sean factores independientes de cosecha ganglionar elevada, aboga también en este sentido.

La mayor sobrevida no está ligada a un efecto terapéutico de la extensión de la resección ${ }^{(8)}$, sino a factores inmunológicos o relación huésped-hospedero.

El trabajo de Markl ${ }^{(51)}$ nos parece excelente para explicar la asociación entre cosecha ganglionar y respuesta inmunológica. Plantea la hipótesis de que el tumor realiza una activación del sistema inmune cuya mayor respuesta tendría como consecuencia un agrandamiento de los ganglios, siendo más fácil su identificación.

En un grupo de 239 pacientes con cáncer de colon y adenopatías negativas, estudia el tamaño de los ganglios como un factor de respuesta inmunológica. Este grupo categoriza a los pacientes de acuerdo a la cantidad de ganglios hallados mayores de $5 \mathrm{~mm}$ (grandes mayores de $5 \mathrm{~mm}$ ).

Los jóvenes tienen mayores conteos de ganglios más grandes. El colon izquierdo tiene conteos más bajos de ganglios grandes. A menor número de ganglios grandes, cosechas ganglionares más pobres. Se objetiva una mayor sobrevida en pacientes con más cantidad de ganglios grandes.

En el momento actual, entonces, se considera que existen causas inmunogénicas y de inmunocompetencia que explican la asociación entre cosecha ganglionar y sobrevida, que ya no puede explicarse solamente por subestadificación o por recaídas locales ${ }^{(6)}$.

\section{Conclusiones}

A pesar de todo lo hablado, sigue siendo esencial no ver lo presentado como una evidencia o argumento a favor de luchar ciegamente por la limitación de la extensión de la resección, o como una sugerencia de que "menos es más" en todas las situaciones. Resecar muy poco es intrínsecamente dañino para los pacientes, y cualquier abordaje conservador de las resecciones de tumores malignos debe seguir una guía muy clara ${ }^{(12)}$.

De todos modos, algunas afirmaciones pueden ser más "mito" que "magia".

- El número 12 no necesariamente es un número "mágico" marcador de calidad, y existen múltiples factores a tener en cuenta.

- Extender la resección para aumentar la cosecha ganglionar no mejora la estadificación y puede exponer al paciente a riesgos innecesarios.

- La linfadenectomía extendida no tiene efecto terapéutico comprobado.

- Menos no es más, pero más no es necesariamente mejor.

La "magia" sigue siendo realizar resecciones regladas, que incluyan el pedículo vascular y el meso satélite al tumor, ajustando la resección a las características del paciente.

El cirujano debe auditar la calidad de sus cirugías y el número 12 es una herramienta de auditoría, pero no la única a ser tenida en cuenta para valorar la calidad de la cirugía.

Si todos los pacientes tienen conteos pobres, seguramente el cirujano deberá revisar su táctica quirúrgica. $\mathrm{Si}$ todos sus conteos son muy altos, seguramente en algunos pacientes estará realizando cirugía innecesaria.

\section{Summary}

Lymph node compromise is critical in colon cancer staging, as a prognostic factor and to determine adjuvant therapy. The number of lymph nodes to be resected is still under discussion, as well as the factor that have an impact on lymph node harvest and its biological significance.

We reviewed clinical variables and variables that are specific to the tumor, what results in the definition of a 
certain number of lymph nodes, as the adequate Gold Standard for lymph node harvest being controversial.

12 is not necessarily a "magic" number that marks quality. Extending resection to increase lymph node harvest does not improve staging, it exposes patients to unnecessary risks, there being no therapeutic effect guaranteed. The "Magic" continues to be routine resection that includes the cystic pedicle and the area around the tumour, adjusting resection to the patient's characteristics. Less is not best, but more is not necessarily better.

\section{Resumo}

O compromisso ganglionar é crítico no estadiamento do câncer de cólon, como fator prognóstico e como determinante do tratamento adjuvante. A discussão sobre o número de gânglios adequados a ressecar, quais são os fatores que incidem sobre a definição do número de linfonodos a ser retirados e seu significado biológico.

Faz-se uma revisão das variáveis clínicas e da própria biologia tumoral, que fazem com que a definição de um número determinado de gânglios como Gold Standard do número adequado de linfonodos a remover seja controversa.

O número 12 não é necessariamente um número "mágico", um marcador de qualidade. Ampliar a ressecção para aumentar o número de linfonodos que serão retirados não melhora o estadiamento, expõe o paciente a riscos desnecessários, sem um efeito terapêutico comprovado.

A "Magia" continua sendo realizar ressecções de acordo com parâmetros definidos, que incluam o pedículo vascular e o mesocólon satélite ao tumor, ajustando a ressecção às características do paciente. Menos não é mais, porém mais não é necessariamente melhor.

\section{Bibliografía}

1. Albatanony A, Alseesi A, Ammar M, Shaaban M. Improving lymph node harvest in colorectal cancer by intra-arterial injection of methylene blue: a randomized trial. Egypt J Surg 2015; 34(2):99-102.

2. Johnson P, Malatjalian D, Porter G. Adequacy of nodal harvest in colorectal cancer: a consecutive cohort study. J Gastrointest Surg 2002; 6(6):883-88.

3. Böckelman C, Engelmann B, Kaprio T, Hansen T, Glimelius B. Risk of recurrence in patients with colon cancer stage II and III: a systematic review and meta-analysis of recent literature. Acta Oncol 2015; 54(1):5-16.

4. Lisovsky M, Schutz S, Drage M, Liu X, Suriawinata A, Srivastava A. Number of lymph nodes in primary nodal basin and a "second look" protocol as quality indicators for optimal nodal staging of colon cancer. Arch Pathol Lab Med 2017; 141(1):125-30.
5. Reha J, Mukkamalla S, Rathore R, Somasundar P. Adequate lymph node evaluation in the elderly is associated with improved survival in patients with stage I-III colon cancer: a validation study using the National Cancer Data Base. Eur J Surg Oncol 2018; 44(1):148-56.

6. Xue L, Williamson A, Gaines S, Andolfi C, Paul-Olson T, Neerukonda A, et al. An update on colorectal cancer. Curr Probl Surg 2018; 55(3):76-116.

7. Resch A, Langner C. Lymph node staging in colorectal cancer: old controversies and recent advances. World J Gastroenterol 2013; 19(46):8515-26.

8. Willaert W, Mareel M, Van De Putte D, Van Nieuwenhove Y, Pattyn P, Ceelen W. Lymphatic spread, nodal count and the extent of lymphadenectomy in cancer of the colon. Cancer Treat Rev 2014; 40(3):405-13

9. Fielding L, Arsenault $\mathbf{P}$, Chapuis $\mathbf{P}$, Dent $\mathbf{O}$, Gathright $\mathbf{B}$, Hardcastle, et al. Clinicopathological staging for colorectal cancer: an International Documentation System (IDS) and an International Comprehensive Anatomical Terminology (ICAT). J Gastroenterol Hepatol 1991; 6(4):325-44.

10. Pickren J. Current concepts in cancer: nodal clearance and detection. JAMA 1975; 231(9):969-71.

11. Scott K, Grace R. Detection of lymph node metastases in colorectal carcinoma before and after fat clearance. Br J Surg 1989; 76(11):1165-7.

12. Amri R, Klos C, Bordeianou L, Berger D. The prognostic value of lymph node ratio in colon cancer is independent of resection length. Am J Surg 2016; 212(2):251-7.

13. Li Destri G, Di Carlo I, Scilletta R, Scilletta B, Puleo S. Colorectal cancer and lymph nodes: the obsession with the number 12. World J Gastroenterol 2014; 20(8):1951-60.

14. Parsons H, Begun J, Kuntz K, Tuttle T, McGovern P, Virnig B. Lymph node evaluation for colon cancer in an era of quality guidelines: who improves? J Oncol Pract 2013; 9(4):e164-71.

15. Backes Y, Elias S, Bhoelan B, Groen J, van Bergeijk J, Seerden $\mathbf{T}$, et al. The prognostic value of lymph node yield in the earliest stage of colorectal cancer: a multicenter cohort study. BMC Med 2017; 15(1):129.

16. Hamza A, Sakhi R, Khawar S, Alrajjal A, Edens J, Khurram M, et al. Role of "second look" lymph node search in harvesting optimal number of lymph nodes for staging of colorectal carcinoma. Gastroenterol Res Pract 2018; 2018:1985031. doi: 10.1155/2018/1985031.

17. Garcia B, Guzman C, Johnson C, Hellenthal N, Monie D, Monzon J. Trends in lymph node excision and impact of positive lymph node ratio in patients with colectomy for primary colon adenocarcinoma: population based study 1988 to 2011. Surg Oncol 2016; 25(3):158-63.

18. Fang S, Efron J, Berho M, Wexner S. Dilemma of stage II colon cancer and decision making for adjuvant chemotherapy. J Am Coll Surg 2014; 219(5):1056-69.

19. Liu J, Huffman K, Palis B, Shulman L, Winchester D, Ko C, Hall B. Reliability of the American College of Surgeons Commission on Cancer's Quality of Care Measures for Hos- 
pital and Surgeon Profiling. J Am Coll Surg 2017; 224(2):180-190.e8. doi: 10.1016/j.jamcollsurg.2016.10.053.

20. Betge J, Harbaum L, Pollheimer M, Lindtner R, Kornprat $\mathbf{P}$, Ebert $\mathbf{M}$, et al. Lymph node retrieval in colorectal cancer: determining factors and prognostic significance. Int J Colorectal Dis 2017; 32(7):991-8.

21. Tekkis P, Smith J, Heriot A, Darzi A, Thompson M, Stamatakis J. A national study on lymph node retrieval in resectional surgery for colorectal cancer. Dis Colon Rectum 2006; 49(11):1673-83.

22. Shen S, Haupt B, Ro J, Zhu J, Bailey H, Schwartz M. Number of lymph nodes examined and associated clinicopathologic factors in colorectal carcinoma. Arch Pathol Lab Med 2009; 133(5):781-6.

23. Wood P, Peirce C, Mulsow J. Non-surgical factors influencing lymph node yield in colon cancer. World J Gastrointest Oncol 2016; 8(5):466-73.

24. Martínez-Ramos D, Escrig-Sos J, Miralles-Tena J, Rivadulla-Serrano I, Salvador-Sanchís J. ¿Existe un número mínimo de ganglios linfáticos que se debe analizar en la cirugía del cáncer colorrectal? Cir Esp 2008; 83(3):108-17.

25. Costa G, Lorenzon L, Massa G, Frezza B, Ferri M, Fransvea $\mathbf{P}$, et al. Emergency surgery for colorectal cancer does not affect nodal harvest comparing elective procedures: a propensity score-matched analysis. Int J Colorectal Dis 2017; 32(10):1453-61.

26. Patel S, Patel S, Brackstone M. Emergency surgery for colorectal cancer does not result in nodal understaging compared with elective surgery. Can J Surg 2014; 57(5):349-53.

27. van Erning F, Crolla R, Rutten H, Beerepoot L, van Krieken $\mathbf{J}$, Lemmens $\mathbf{V}$. No change in lymph node positivity rate despite increased lymph node yield and improved survival in colon cancer. Eur J Cancer 2014; 50(18):3221-9.

28. Fingerhut A. What counts most in the lymph node count for colorectal cancer? Surg Innov 2012; 19(3):213-5.

29. Le Voyer T, Sigurdson E, Hanlon A, Mayer R, Macdonald $\mathbf{J}$, Catalano $\mathbf{P}$, et al. Colon cancer survival is associated with increasing number of lymph nodes analyzed: a secondary survey of intergroup trial INT-0089. J Clin Oncol 2003; 21(15):2912-9.

30. Tsai H, Huang C, Yeh Y, Ma C, Chen C, Lu C, et al. Factors affecting number of lymph nodes harvested and the impact of examining a minimum of 12 lymph nodes in stage I-III colorectal cancer patients: a retrospective single institution cohort study of 1167 consecutive patients. BMC Surg 2016; $16: 17$.

31. Edler D, Ohrling K, Hallström M, Karlberg M, Ragnhammar P. The number of analyzed lymph nodes - a prognostic factor in colorectal cancer. Acta Oncol 2007; 46(7):975-81.

32. Chang G, Rodriguez-Bigas M, Skibber J, Moyer V. Lymph node evaluation and survival after curative resection of colon cancer: systematic review. J Natl Cancer Inst 2007; 99(6):433-41
33. Törnroos A, Garvin S, Olsson H. The number of identified lymph node metastases increases continuously with increased total lymph node recovery in pT3 colon cancer. Acta Oncol 2009; 48(8):1152-6.

34. Törnroos A, Shabo I, Druvefors B, Arbman G, Olsson H. Postoperative intra-arterial methylene blue injection of colorectal cancer specimens increases the number of lymph nodes recovered. Histopathology 2011; 58(3):408-13.

35. Choi H, Law W, Poon J. The optimal number of lymph nodes examined in stage II colorectal cancer and its impact of on outcomes. BMC Cancer 2010; 10:267.

36. O'Boyle S, Stephenson K. More is better: lymph node harvesting in colorectal cancer. Am J Surg 2017; 213(5):926-30.

37. Gravante G, Parker R, Elshaer M, Mogekwu A, Humayun N, Thomas K, et al. Lymph node retrieval for colorectal cancer: Estimation of the minimum resection length to achieve at least 12 lymph nodes for the pathological analysis. Int J Surg 2016; 25:153-7.

38. Stracci F, Bianconi F, Leite S, Liso A, La Rosa F, Lancellotta $\mathrm{V}$, et al. Linking surgical specimen length and examined lymph nodes in colorectal cancer patients. Eur J Surg Oncol 2016; 42(2):260-5

39. Khan H, Olszewski A, Somasundar P. Lymph node involvement in colon cancer patients decreases with age; a population based analysis. Eur J Surg Oncol 2014; 40(11):1474-80.

40. Lee L, Erkan A, Alhassan N, Kelly J, Nassif G, Albert M, et al. Lower survival after right-sided versus left-sided colon cancers: Is an extended lymphadenectomy the answer? Surg Oncol 2018; 27(3):449-55.

41. Parsons H, Tuttle T, Kuntz K, Begun J, McGovern P, Virnig B. Association between lymph node evaluation for colon cancer and node positivity over the past 20 years. JAMA 2011; 306(10):1089-97.

42. Budde C, Tsikitis V, Deveney K, Diggs B, Lu K, Herzig D. Increasing the number of lymph nodes examined after colectomy does not improve colon cancer staging. J Am Coll Surg 2014; 218(5):1004-11.

43. Del Paggio J, Nanji S, Wei X, MacDonald P, Booth C. Lymph node evaluation for colon cancer in routine clinical practice: a population-based study. Curr Oncol 2017; 24(1):e35-e43. doi: 10.3747/co.24.3210

44. Kawamura Y, Umetani N, Sunami E, Watanabe T, Masaki T, Muto T. Effect of high ligation on the long-term result of patients with operable colon cancer, particularly those with limited nodal involvement. Eur J Surg 2000; 166(10):803-7.

45. Canessa C, Badía F, Fierro S, Fiol V, Háyek G. Anatomic study of the lymph nodes of the mesorectum. Dis Colon Rectum 2001; 44(9):1333-6.

46. Hashiguchi Y, Hase K, Ueno H, Mochizuki H, Shinto E, Yamamoto J. Optimal margins and lymphadenectomy in colonic cancer surgery. Br J Surg 2011; 98(8):1171-8.

47. Bertelsen C, Neuenschwander A, Jansen J, Kirkegaard-Klitbo A, Tenma J, Wilhelmsen M, et al. Short-term outcomes after complete mesocolic excision compared with 
'conventional' colonic cancer surgery. Br J Surg 2016; 103(5):581-9.

48. Fan Y, Ning F, Zhang C, Dai D. Preservation versus non-preservation of left colic artery in sigmoid and rectal cancer surgery: a meta-analysis. Int J Surg 2018; 52:269-77.

49. Paquette I, Madoff R, Sigurdson E, Chang G. Impact of proximal vascular ligation on survival of patients with colon cancer. Ann Surg Oncol 2018; 25(1):38-45.
50. Manceau G, Cotte E. Colectomie angulaire gauche vs colectomie subtotale dans les cancers de l'angle colique gauche. Colon Rectum 2017; 11:259-63.

51. Märkl B, Schaller T, Kokot Y, Endhardt K, Kretsinger H, Hirschbühl K, et al. Lymph node size as a simple prognostic factor in node negative colon cancer and an alternative thesis to stage migration. Am J Surg 2016; 212(4):775-80.

\section{Contribución de autores}

Ricardo Misa, https://orcid.org/0000-0002-8282-6863. Autor principal, concepción, diseño, ejecución, análisis, interpretación de resultados, redacción, revisión.

Mario Almada, https://orcid.org/0000-0002-4655-2789. Ejecución, análisis, interpretación de resultados.

Noelia Brito, https://orcid.org/0000-0002-1394-3994. Ejecución, análisis, interpretación de resultados.

José Martínez, https://orcid.org/0000-0001-8234-228X. Ejecución, análisis.

Andrés Pouy, https://orcid.org/0000-0002-7136-144X. Ejecución, redacción, interpretación de resultados.

Camila Haro, https://orcid.org/0000-0003-4475-9740. Ejecución, interpretación de resultados. 